\title{
Numerical study of the flow inside an alternator: application to the thermal optimization of the machine
}

\author{
P. O. Jandaud ${ }^{1,2}$, S. Harmand ${ }^{1} \&$ M. Fakes ${ }^{2}$ \\ ${ }^{1}$ Université Lille Nord de France, Valenciennes, France \\ ${ }^{2}$ Valeo Electrical Systems, France
}

\begin{abstract}
In this paper, the flow inside an electric machine called a starter-alternator is studied parametrically with CFD in order to be used by a thermal lumped model coupled to an optimization algorithm using Particle Swarm Optimization (PSO). In the first case, the geometrical parameters are symmetric allowing us to model only one side of the machine. The optimized thermal results are not conclusive. In the second case, all the parameters are independent. In this case, the flow is strongly influenced by the dissymmetry. Optimization results are this time a clear improvement compared to the original machine.
\end{abstract}

Keywords: CFD, lumped method, electric machine, optimization, PSO.

\section{Introduction}

In order to reduce $\mathrm{CO}_{2}$ emissions and gas consumptions, the automotive industry is developing more and more hybrid vehicles. Therefore, it needs to create innovative technologies. For mild-hybrid cars, a combustion engine is still necessary but it is coupled to an electric machine called starter-alternator. This machine is powerful, the electric power produced is around $10 \mathrm{~kW}$, and compact, its diameter is approximately $20 \mathrm{~cm}$ and its length $10 \mathrm{~cm}$. The cooling of the starter-alternator is a critical point in its design as high temperatures affect the performances of the machine.

To study the thermal behaviour of electric machines, a code named SAME (Aero-thermal Simulation of Electric Machines) has been developed at the laboratory. The code uses lumped method to create a network of thermal conductances and can be coupled to optimization algorithm. This method is 
widely used due as it does not need a lot of computational resources compared to CFD (Boglietti et al. [1]) and the results accuracy is not far from CFD if the model is well built. In our case, SAME uses a fine discretization of the machine and needs a precise description of the flow distribution inside. It has been obtained using CFD with a RANS model.

The final objective of this study is to obtain an optimal geometry from a thermal point view. The optimization parameters retained for the study are only geometrical parameters which do not interact with the electric performances of the machine. The optimization part is done with the Particle Swarm Optimization (PSO) algorithm created by Kennedy and Eberhart [2] in 1995. This algorithm is used in a wide variety of fields (Poli [3]). In thermal engineering, Genetic Algorithm (GA) is the most used optimization tool (Gosselin et al. [4]) but PSO, which has better performances than GA, is more and more used for heat transfer problems like in the study by Bornshlegell et al. [5]. To be able to optimize the machine, the flow distribution inside has to be known for every geometrical configuration possible within the optimization space.

In this paper, a parametric study of the flow inside the starter-alternator is presented. CFD computations have been made for different geometrical configurations and results have been interpolated in order to have a continuous function in the optimization space. The consequences on the thermal behaviour of the machine and the optimization results are shown.

\section{Models presentation}

\subsection{Machine overview}

The starter-alternator (figure 1) is an electric machine constituted of cylindrical rotor and stator. Two centrifugal fans are situated at each side of the rotor. The machine is enclosed by two brackets (front and rear) with inlets and outlets allowing the flow to come in and out. The flow enters the machine axially

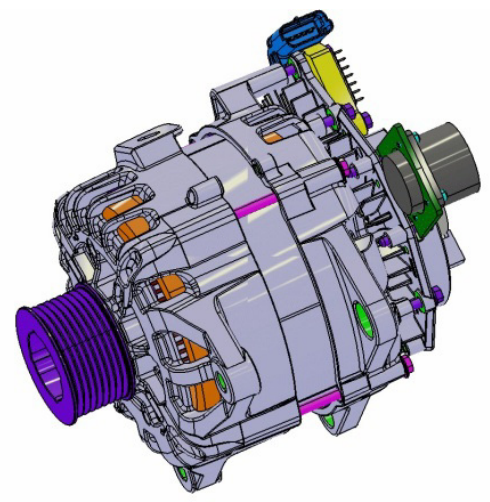

Figure 1: $\quad$ External view of the machine. 
through inlets and exits radially through outlets (figure 2). A part of the flow goes from the front $\left(\mathrm{Q}_{\text {front }}\right)$ to the rear $\left(\mathrm{Q}_{\text {rear }}\right)$ through the air gap between stator and rotor $\left(\mathrm{Q}_{\text {gap }}\right)$ and through rotor channels $\left(\mathrm{Q}_{\mathrm{rot}}\right)$.

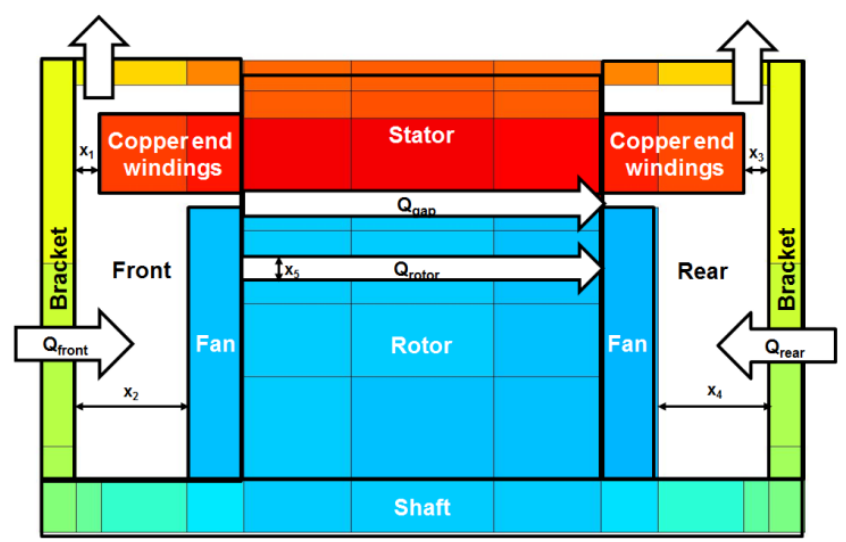

Figure 2: $\quad$ Architecture, flow distribution and optimization parameters.

The geometrical parameters chosen for optimization are (figure 2): gaps between windings and brackets $\left(\mathrm{x}_{1}\right.$ and $\left.\mathrm{x}_{3}\right)$, gaps between fans and brackets $\left(\mathrm{x}_{2}\right.$ and $\left.\mathrm{x}_{4}\right)$ and the size of the rotor channels $\left(\mathrm{x}_{5}\right)$. All the $\mathrm{x}_{\mathrm{i}}$ values are grouped in a single vector $\mathrm{x}$ called optimization vector.

All these parameters are normalized using equation (1) for geometrical parameters and equation (2) for volume flow rates. In equation (2), absolute value of the value is taken because the direction of the flow inside the rotor and the air gap can change with the different geometrical configurations.

$$
\begin{gathered}
x_{i}^{*}=\frac{x_{i}-\min \left(x_{i}\right)}{\max \left(x_{i}\right)-\min \left(x_{i}\right)} \\
Q_{\text {zone }}^{*}=\frac{\left|Q_{\text {zone }}\right|-\min \left(\left|Q_{\text {zone }}\right|\right)}{\max \left(\left|Q_{\text {zone }}\right|\right)-\min \left(\left|Q_{\text {zone }}\right|\right)}
\end{gathered}
$$

The reference machine has symmetry between front and rear and $x^{*}=[0.17$; $1 ; 0.17 ; 1 ; 0]$. In this machine, the rotor channels are totally closed.

\subsection{Thermal model}

The model used here is the lumped method (Glises [6]). The domain is divided in small volumes considered isothermal. Heat transfers between all volumes are modelled by a network of thermal conductances. These conductances have different expressions depending on the nature of the heat transfer: conduction, convection and inside the fluid. They are calculated the distance between two cells, their contact surface, thermal conductivity, convective heat transfer 
coefficient and mass flow rate. The convective heat transfer coefficient $(\mathrm{h})$ is difficult to obtain because it depends strongly on the geometry. To solve this problem, at each contact between a fluid cell and a solid cell, the code considers a simpler and more theoretical geometry. For example, for the flow parallel to the rotor, a correlation giving $\mathrm{h}$ for a parallel flow on a rotating disk (Wiesche [7]) is used.

Using this method, heat equation can be simplified into a simple matrix equation (3) even simpler in steady state (4). C is the matrix of conductivity, $\mathrm{T}$ vector of temperatures, G matrix of conductances and $\mathrm{P}$ vector of heat losses.

$$
\begin{gathered}
C \frac{d T}{d t}=G T+P \\
G T=-P
\end{gathered}
$$

For the rest of the paper, we will only consider temperature differences with the temperatures of the reference case:

$$
\Delta \mathrm{T}=T-T_{\text {ref }}
$$

\subsection{CFD model}

In order to calculate fluid and convective conductances, the code needs the flow distribution inside the machine. For lumped method, a head losses model seems to be a natural choice given the fact the two methods have a global approach. For example, they are coupled in [5]. In our case, as we have several inlets and outlets as well as we do not know exactly the flow direction in certain zones of the machine, CFD is used.

Calculations are realized with Fluent 13 , with a geometry directly imported from simplified CAD drawings. Reynolds Averaged Navier-Stokes equations are solved using the k- $\varepsilon$ realisable model. Standard wall functions are used to solve the turbulent boundary layer to be able to have a coarser mesh. We have a $\mathrm{y}^{+}$ between 1 and 10 . In order to use optimization, the mesh is entirely parameterised with the geometrical parameters of figure 2 .

For the study, two cases are considered. In the first one, geometry is considered symmetric between front and rear. As a result, only the front of the machine is modelled and the only two parameters used are $\mathrm{x}_{1}$ and $\mathrm{x}_{2}$. The only volume flow rate calculated is $\mathrm{Q}_{\text {front }}$. Computations are done for several values of are $x_{1}$ and $x_{2}$ given in equations (6) and (7) giving a total of 72 different simulations. Results are then interpolated to obtain a continuous function of $\mathrm{x}_{1}$ and $\mathrm{x}_{2}$ for $\mathrm{Q}_{\text {front }}$.

$$
\begin{aligned}
& x_{1}^{*}=\left[\begin{array}{llllllllll}
0 & 0.1 & 0.2 & 0.3 & 0.4 & 0.5 & 0.6 & 0.7 & 0.8 & 0.9
\end{array}\right] \\
& x_{2}^{*}=\left[\begin{array}{llllll}
0 & 0.2 & 0.4 & 0.6 & 0.8 & 1
\end{array}\right]
\end{aligned}
$$


The second case has five dimensions: the machine is entirely modelled. The hypercube defined by the parameters $x_{i}^{*}$ is discretized more coarsely. The different values used for the simulations are given in equations (8), (9), (10), (11) and (12) giving 256 different possible combinations. Results are interpolated to have the complete flow distribution inside the machine function of these five parameters.

$$
\begin{aligned}
& x_{1}^{*}=\left[\begin{array}{llll}
0 & 0.3 & 0.6 & 1
\end{array}\right] \\
& x_{2}^{*}=\left[\begin{array}{ll}
0 & 1
\end{array}\right] \\
& x_{3}^{*}=\left[\begin{array}{llll}
0 & 0.3 & 0.6 & 1
\end{array}\right] \\
& x_{4}^{*}=\left[\begin{array}{ll}
0 & 1
\end{array}\right] \\
& x_{5}^{*}=\left[\begin{array}{llll}
0 & 0.4 & 0.7 & 1
\end{array}\right]
\end{aligned}
$$

\subsection{Optimization algorithm}

The optimization method coupled to our thermal model is Particle Swarm Optimization. PSO is a relatively new optimization method created by Kennedy and Eberhart in 1995 [2]. The principle is based on animals Swarm Behaviour. In this algorithm, the population is a swarm of particle. Each particle knows its current position, its best position and the best position of the swarm. At each generation, each particle moves accordingly to a velocity vector based on these three parameters.

In the algorithm, each particle $i$ has two associated vectors: its position $x_{i}$ and velocity $v_{i}$. For a generation $n$, the position of the particle $x_{i}(n)$ depends on its velocity $v_{i}(n)$, the best position of the swarm $g$ and its best known position $p_{i}$. We have then:

$$
\begin{gathered}
v_{i}(n)=\omega \cdot v_{i}(n-1)+\varphi_{p} \cdot r_{p}\left(p_{i}-x_{i}(n-1)\right) \\
+\varphi_{g} \cdot r_{g}\left(g-x_{i}(n-1)\right) \\
x_{i}(n)=x_{i}(n-1)+v_{i}(n) \\
\text { And if } f\left(x_{i}\right)<f\left(p_{i}\right) \text { then } p_{i}=x_{i} \\
\text { And if } f\left(x_{i}\right)<f(g) \text { then } g=x_{i}
\end{gathered}
$$

$\omega, \varphi_{p}, \varphi_{g}$ are predefined constants.

$r_{p}, r_{g}$ are random number taken on $[0 ; 1]$.

$f$ is the objective function.

The algorithm continues from (13) to (16) until a stopping criterion is met. 


\section{Results and analysis}

\subsection{Symmetric case}

\subsubsection{Flow analysis}

The results in figure 3 show the volume flow rate at the front $Q_{\text {front }}^{*}$ function of $x_{1}^{*}$ and $x_{2}^{*}$ respectively the gap between the copper end windings and the bracket and the gap fan/bracket. The graph shows $x_{2}^{*}$ does not have a strong influence on the flow. The flow is maximal for $x_{2}^{*}=0$ and minimal for $x_{2}^{*}=1$. $x_{1}^{*}$ has a lot more influence on the flow. The flow rate is monotonically increasing with $x_{1}^{*}$. For $0<x_{1}^{*}<0.5$, it increases strongly and for $0.5<x_{1}^{*}<1$, it slowly converges towards the maximum. The absolute maximum of the function is located in $x^{*}=\left[\begin{array}{ll}1 & 0\end{array}\right]$.

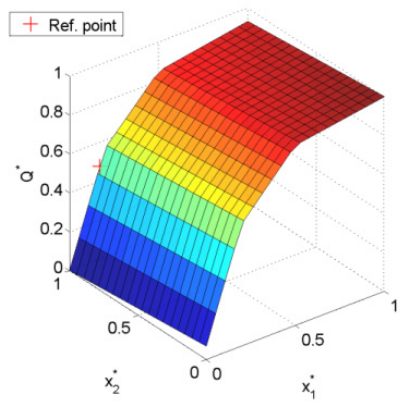

Figure 3: $\quad$ Surface of $Q_{\text {front }}^{*}$ for the symmetric case.

\subsubsection{Thermal results}

In figure 4 , the results show the evolutions of the mean temperature inside the rotor and the stator. The most noticeably remark is the temperature minima do not correspond to the volume flow rate maximum. For convective heat transfer, the two important parameters are the convective heat transfer coefficient $(h)$ and
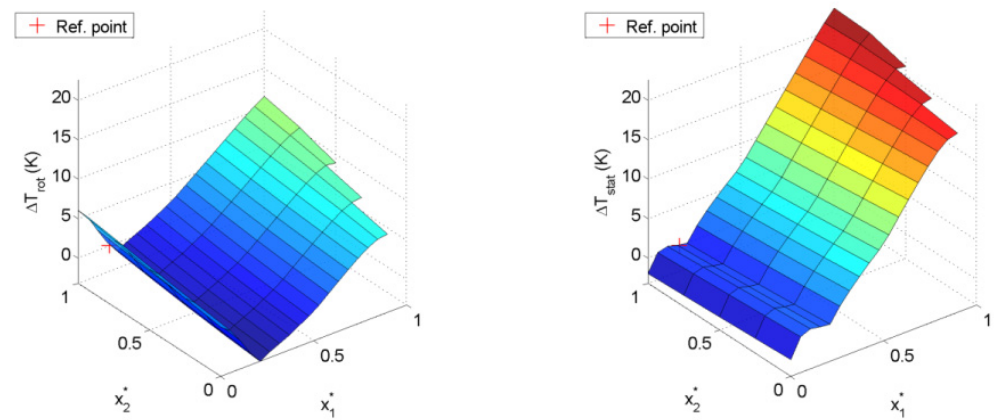

Figure 4: $\quad$ Surfaces of $\Delta T_{\text {rot }}$ and $\Delta T_{\text {stat }}$ for the symmetric case. 
the contact surface between fluid and solid (S). When $x_{1}^{*}$ increases and $x_{2}^{*}$ decreases, the velocity inside the machine increases and, as a result, so does $h$. But, in the meantime, $\mathrm{S}$ is decreasing. The minima of temperatures are where the product of $\mathrm{h}$ and $\mathrm{S}$ is maximal. To find them, optimization is used.

\subsubsection{Optimization results}

PSO is used to solve the optimization problem. The optimization space is the square $[0 ; 1]^{2}$. Two different objective functions are chose: average temperature inside the rotor and inside the stator. Results are presented in table 1. First, the results show different minima depending on the objective. The objectives give two almost opposite configurations improving the goal set but degrading the other objective. Second, the optima do not improve significantly the reference case: the reference machine is well designed and can't be really improve if the evolution of geometrical parameters stays symmetric.

Table 1: Optimization results for the symmetric case.

\begin{tabular}{|c|c|c|c|c|}
\hline Case & $x_{1}^{*}$ & $x_{2}^{*}$ & $\Delta T_{\text {rot }}$ & $\Delta T_{\text {stat }}$ \\
\hline Reference & 0.17 & 1 & 0 & 0 \\
\hline Rotor & 0.21 & 0 & -3.2 & 1.5 \\
\hline Stator & 0 & 0.2 & 6 & -2 \\
\hline
\end{tabular}

\subsection{Asymmetric case}

In this case, all the $x_{i}^{*}$ parameters are evolving independently.

\subsubsection{Flow analysis}

The effect of dissymmetry between $x_{1}^{*}, x_{2}^{*}, x_{3}^{*}$ and $x_{4}^{*}$ are shown in figure 5 . The influence of the dissymmetry between $x_{1}^{*}$ and $x_{3}^{*}$ is strong. For example, at the front, for low $x_{1}^{*}$, the flow rate increases by 0.2 with $x_{3}^{*}$. The opposite happens at the rear. Fans positions ( $x_{2}^{*}$ and $x_{4}^{*}$ ) have less influence on the front. At the rear, the change of fans configuration can change the flow rate by 0.2 .

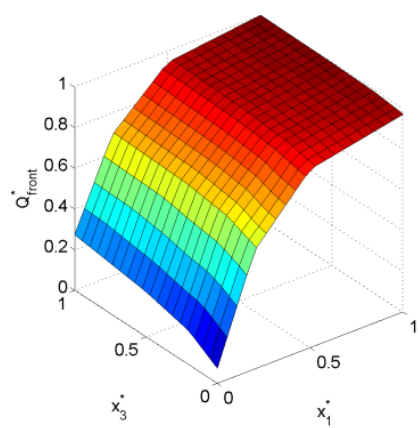

(a) $Q_{\text {front }}^{*}$ evolution for $x_{2}^{*}=0$, $x_{4}^{*}=0, x_{5}^{*}=1$

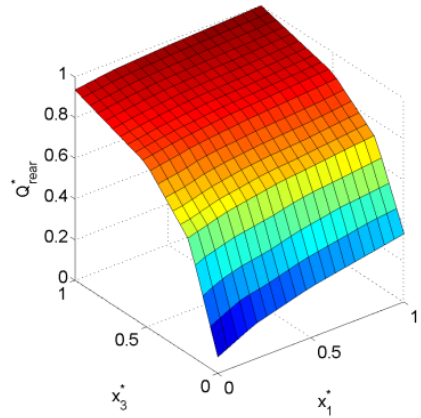

(b) $Q_{\text {rear }}^{*}$ evolution for $x_{2}^{*}=0$, $x_{4}^{*}=0, x_{5}^{*}=1$

Figure 5: Distribution of flow inside the machine in the asymmetric case. 

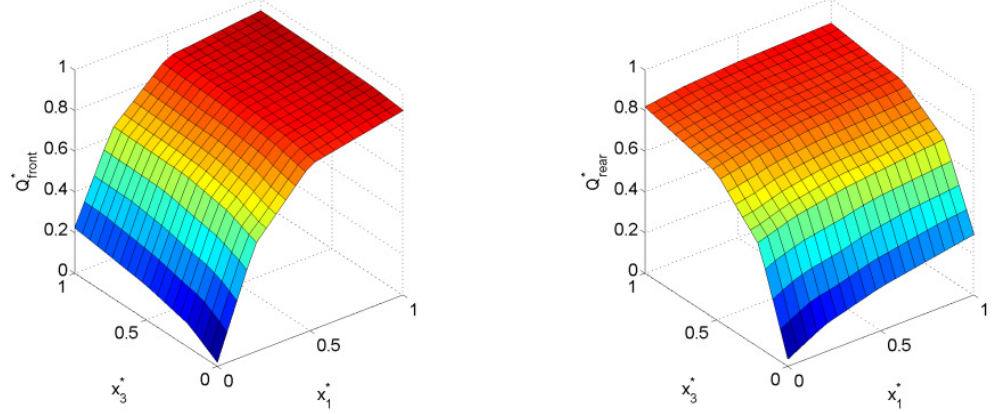

(c) $Q_{\text {front }}^{*}$ evolution for $x_{2}^{*}=0$,

(d) $Q_{\text {rear }}^{*}$ evolution for $x_{2}^{*}=0$,

$x_{4}^{*}=1, x_{5}^{*}=1$

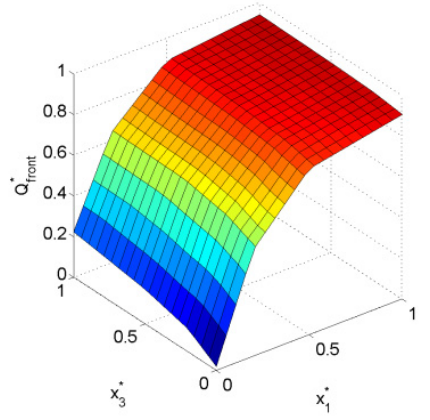

$$
x_{4}^{*}=1, x_{5}^{*}=1
$$

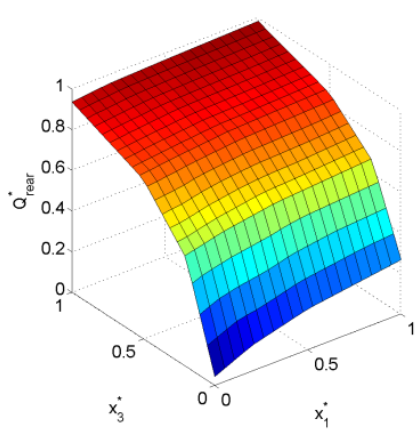

(e) $Q_{\text {front }}^{*}$ evolution for $x_{2}^{*}=1$,

(f) $Q_{\text {rear }}^{*}$ evolution for $x_{2}^{*}=1$,

$$
x_{4}^{*}=0, x_{5}^{*}=1
$$

$$
x_{4}^{*}=0, x_{5}^{*}=1
$$
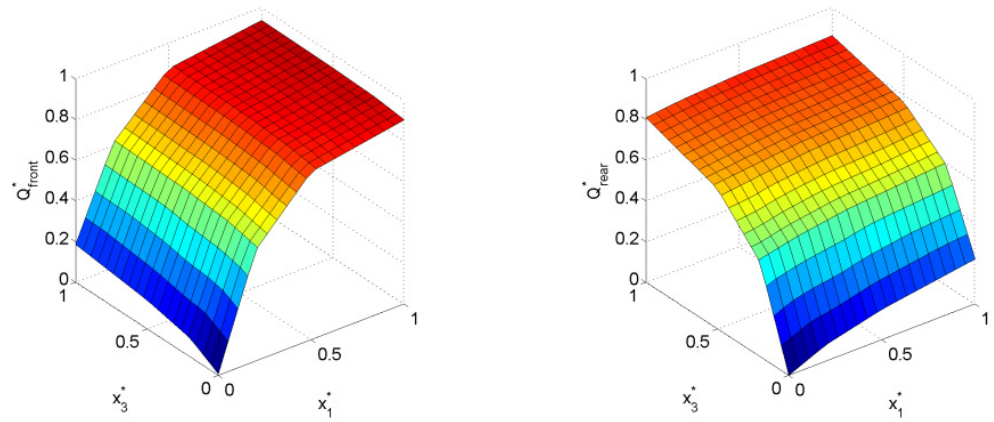

(g) $Q_{\text {front }}^{*}$ evolution for $x_{2}^{*}=1$,

$$
x_{4}^{*}=1, x_{5}^{*}=1
$$

(h) $Q_{\text {rear }}^{*}$ evolution for $x_{2}^{*}=1$, $x_{4}^{*}=1, x_{5}^{*}=1$

Figure 5: Continued.

Another parameter, the rotor channel size $\left(x_{5}^{*}\right)$, has a strong influence on flow, especially the flow inside the rotor and the air gap. Figure 6 shows the evolution, of these two flow rates when $x_{5}^{*}$ is increasing. First, in the air gap, the flow rate 


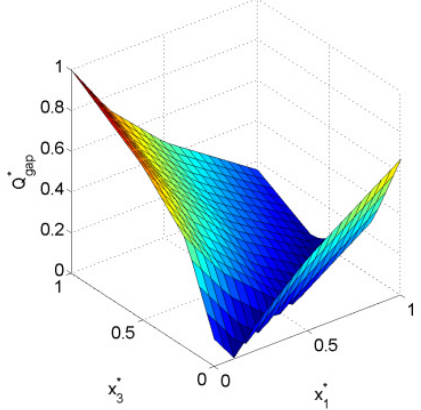

(a) $Q_{\text {gap }}^{*}$ evolution for $x_{2}^{*}=0$, $x_{4}^{*}=0, x_{5}^{*}=0$

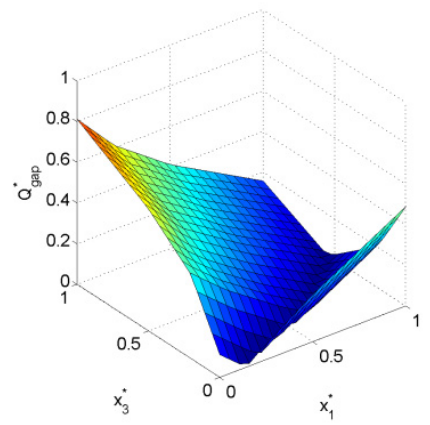

(c) $Q_{\text {gap }}^{*}$ evolution for $x_{2}^{*}=0$, $x_{4}^{*}=0, x_{5}^{*}=0.5$

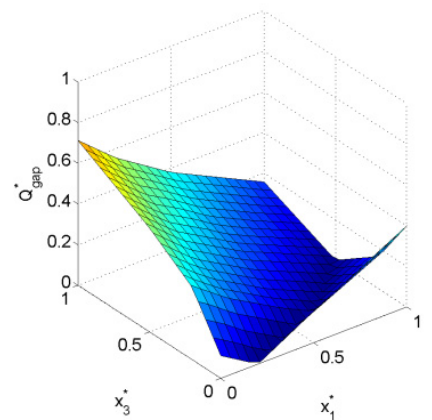

(e) $Q_{\text {gap }}^{*}$ evolution for $x_{2}^{*}=0$,

$$
x_{4}^{*}=0, x_{5}^{*}=1
$$

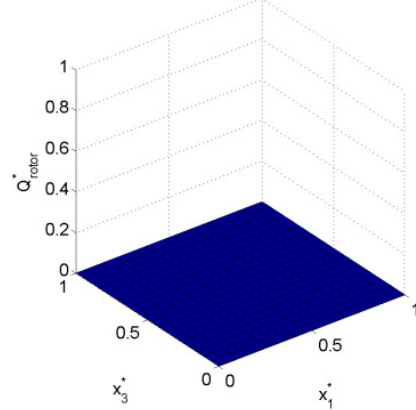

(b) $Q_{\text {rotor }}^{*}$ evolution for $x_{2}^{*}=0$, $x_{4}^{*}=0, x_{5}^{*}=0$

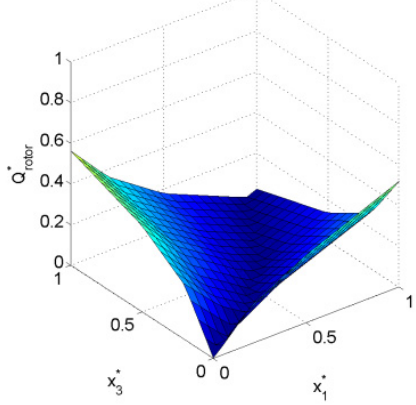

(d) $Q_{\text {rotor }}^{*}$ evolution for $x_{2}^{*}=0$, $x_{4}^{*}=0, x_{5}^{*}=0.5$

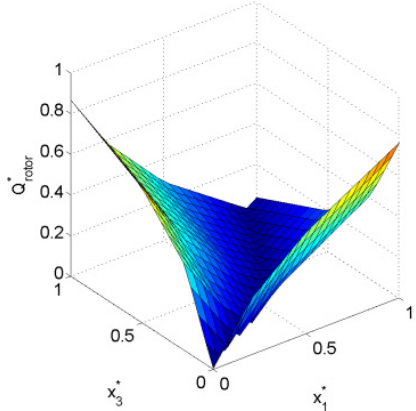

(f) $Q_{\text {rotor }}^{*}$ evolution for $x_{2}^{*}=0$, $x_{4}^{*}=0, x_{5}^{*}=1$

Figure 6: Distribution of flow inside the air gap and the rotor channels.

decreases significantly when the channels open. In the meantime, the flow inside these channels increases. On each sub-figures, the flow changes its direction when $Q^{*}=0$. It can be interesting to control it to force the flow in certain parts 
of the machine. Last, in the air gap, the flow seems much more influenced by $x_{3}^{*}$. In the rotor, the flow is equally influenced by $x_{1}^{*}$ and $x_{3}^{*}$.

\subsubsection{Thermal and optimization results}

Given the fact that temperature evolves in five dimensions, no direct visualization can be made. In this case, optimization shows really its interest. The results are given in table 2 .

Table 2: $\quad$ Optimization results for asymmetric case.

\begin{tabular}{|c|c|c|c|c|c|c|c|}
\hline Case & $x_{1}^{*}$ & $x_{2}^{*}$ & $x_{3}^{*}$ & $x_{4}^{*}$ & $x_{5}^{*}$ & $\Delta T_{\text {rot }}$ & $\Delta T_{\text {stat }}$ \\
\hline Reference & 0.17 & 1 & 0.17 & 1 & 0 & 0 & 0 \\
\hline Sym. Rotor & 0.21 & 0 & 0.21 & 0 & 0 & $\mathbf{- 3 . 2}$ & 1.5 \\
\hline Sym. Stator & 0 & 0.20 & 0 & 0.2 & 0 & 6.0 & $\mathbf{- 2 . 0}$ \\
\hline Rotor & 0.11 & 0.68 & 0.93 & 0.16 & 1 & $\mathbf{- 7 0 . 9}$ & -3.1 \\
\hline Stator & 0 & 0.3 & 0.18 & 0.75 & 0.94 & $\mathbf{- 5 0 . 5}$ & $\mathbf{- 9 . 5}$ \\
\hline
\end{tabular}

The configurations obtained are completely asymmetric. For the case where the rotor is minimized, the sizes of the copper end windings are completely opposite as well as the fans positions. For the stator temperature case, the gaps between the brackets and the copper end windings stay small on both sides for the optimal solution. In the stator, heat is mainly dissipated by convection around the windings: the bigger the exchange surface is, the better the cooling will be.

Contrary to the symmetric, optimal results in both cases are also improving the other objective. Moreover, adding the rotor channels change drastically the temperature levels inside the rotor.

\section{Conclusions}

In this article, we made a parametric study of the flow inside an electric machine called starter-alternator using CFD. First, the geometrical parameters were considered symmetric allowing us to model only one side of the machine. Second, the parameters were considered independent: the complete machine had to be modeled. In this case, the flow is strongly influenced by the dissymmetry.

The flow results were then used in a thermal lumped model of the machine coupled to an optimization tool using Particle Swarm Optimization. In the symmetric case, the possible improvements to the machine are very limited. The asymmetry has then to be used. In this case, optimization results are far better showing a real improvement of the machine. The optimal configurations are completely dissymmetric showing new architectural solutions for electric machines. These solutions improves highly their cooling and, as a result, their electric power. 


\section{Acknowledgements}

This work has been realized as a part of ADEME project MHYGALE. The authors are grateful for the financial support provided by the ADEME, Valeo and the MEDEE project.

\section{References}

[1] Boglietti A., Cavagnino A., Staton D., Shanel M., Mueller M., Mejuto C. Evolution and Modern Approaches for Thermal Analysis of Electrical Machines. Industrial Electronics, IEEE Transactions on. 56-3. 871-882

[2] Kennedy J., Eberhart R. Particle Swarm Optimization, Proceedings of IEEE International Conference on Neural Networks IV, 1942-1948 (1995).

[3] Poli R. Analysis of the publications on the applications of particle swarm optimization, Journal of Artificial Evolution and Applications 2008, 1-10 (2008).

[4] Gosselin L., Tye-Gingras M., Mathieu-Potvin F. Review of utilization of genetic algorithms in heat transfer problems, International Journal of Heat and Mass Transfer, 52 (9-10), 2169-2188 (2009).

[5] Bornschlegell A., Pellé J., Harmand S., Bekrar A., Chaabane S., Trentesaux D. Thermal Optimization of a Single Inlet T-junction, International Journal of Thermal Sciences 53 108-118 (2012).

[6] Glises R. Machines électriques tournantes, Techniques de l'ingénieur, Ref. D3760

[7] aus der Wiesche S. Heat transfer from a rotating disk in a parallel air crossflow. International Journal of Thermal Sciences, vol. 46, no. 8, 745-754 (2007). 